\title{
LE CONGRÉS INTERNATIONAL D'ÉLECTRICITÉ DE GRENOBLE
}

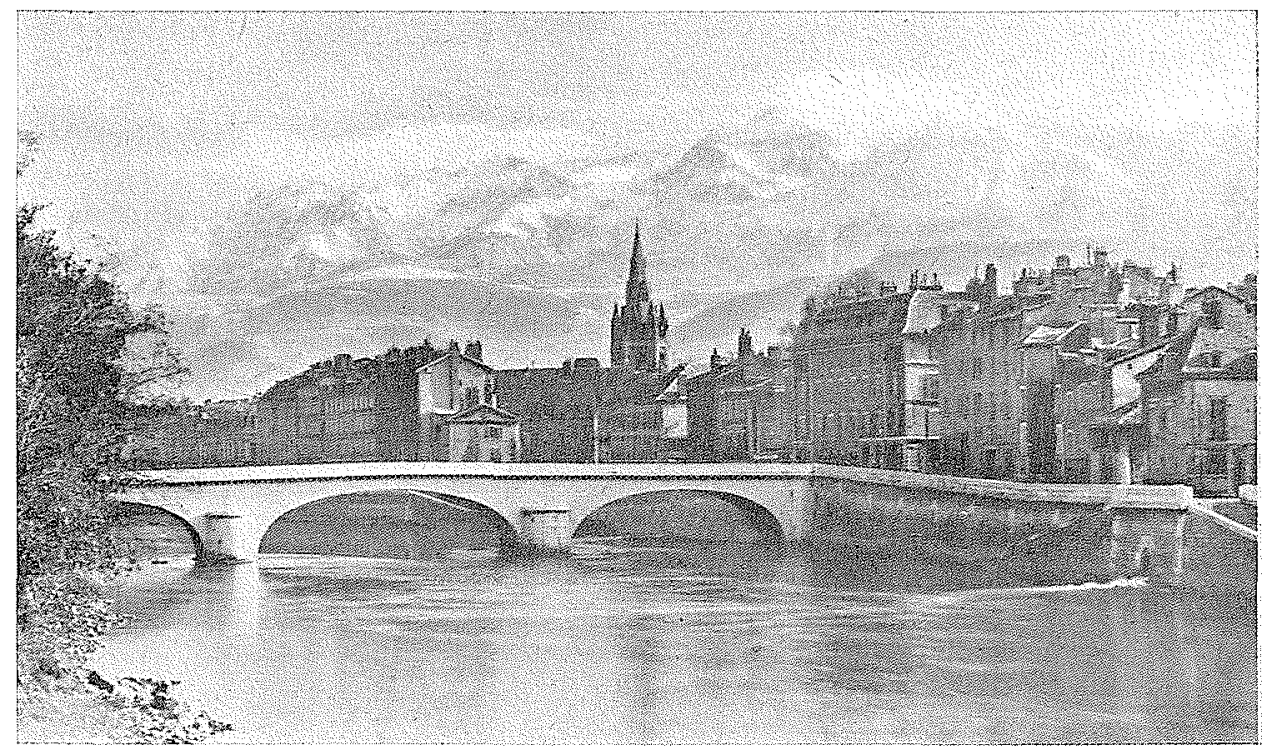

Photo Odions

La Conférence Internationale des Grands Réseaux Electriques a célébré, cette année, le $25^{\circ}$ anniversaire de sa fondation.

Fondée en 1921, la C.I.G.R.E. a pour objet de découvirir et de diffuser les progiès de l'électrotechnique en réunissant périodiquement les électriciens de tous pays. Aussi, après sept ans d'interruption, la session de 1946, tenue à Paris du 27 juin au 7 juillet, a présenté un caractère exceptionnel de documentation, tant par la présentation et la discussion des rapports proposés par les techniciens des différents pays, que par les visites organisées, qui ont permis aux étrangers de voir les réalisations accomplies en France depuis 1939 .

Faisant suite à la Conférence des Grands Réseaux, un important Congrès international d'électricité a eu lieu à Grenoble du 8 au 11 juillet à l'occasion de la $13^{\circ}$ Foire de Grenoble, sous le patronage de la Société Française des Electriciens et la présidence eflective de M. F. Esclangon, Directeur de l'Institut Polytechnique. M. Guy Lorrs, Ingénieur du Centre National de la Recherche Scientifique, promoteur du Congrès ef Commissaire général, en assura l'organisation.
Alors que la $11^{\prime \prime}$ session de la C.I.G.R.E. avait un programme très vaste de conférences, le Congrès de Grenoble a réservé une large place aux visites d'usines et d'installations, dont la région est particulièrement riche.

La découverte de la force hydraulique des torrents à la fin du siecle dernier contribua, pour une grande part, à la fortune de Grenoble. La mélallurgie s’est adaptée aux nouvelles fabrications de turbines, conduites forcées, gros matériel électrique pour l'équipement des centrales hydroélectriques. D'autre part, d'importants laboratoires de recherches se sont créés, mettant à la disposition des réalisateurs, leurs bureaux d'études et leurs stations d'essais. Enfin, disons que Grenoble, capitale de la « Houille Blanche », possède un Institut Polytechnique qui forme, chaque année, dans son Institut d'Electrotechnique (I.E.G.) et son Ecole des Ingénieurs Hydrauliciens (E.I.H.), des techniciens très recherchés.

Le Congrès, dont nous voulons donner dans cet article une vue générale, nous réservant de publier à part quelques conférences, a été ouvert le 8 juillet par M. Hasgetsen, Inspecteur général des Ponts et Chaussés, Chef de la $6^{\circ}$ circoncrip- 


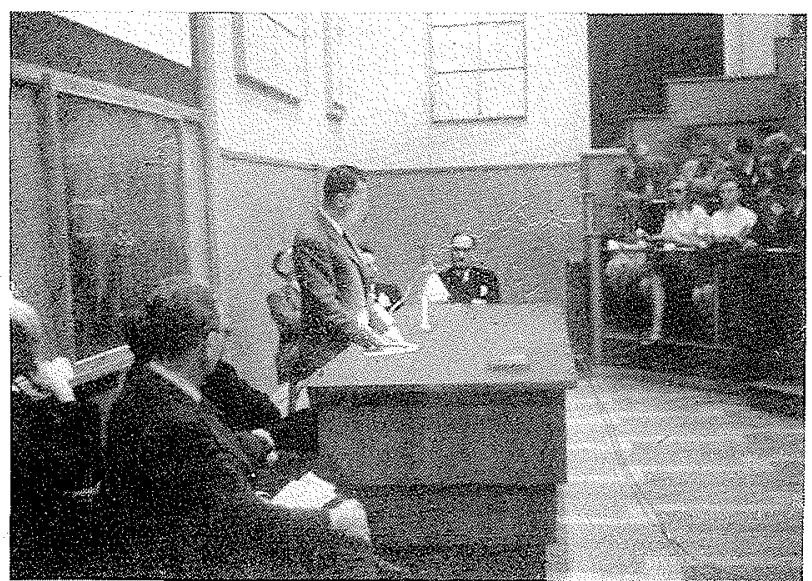

M. HENTY PENDANT SON EXPOSE

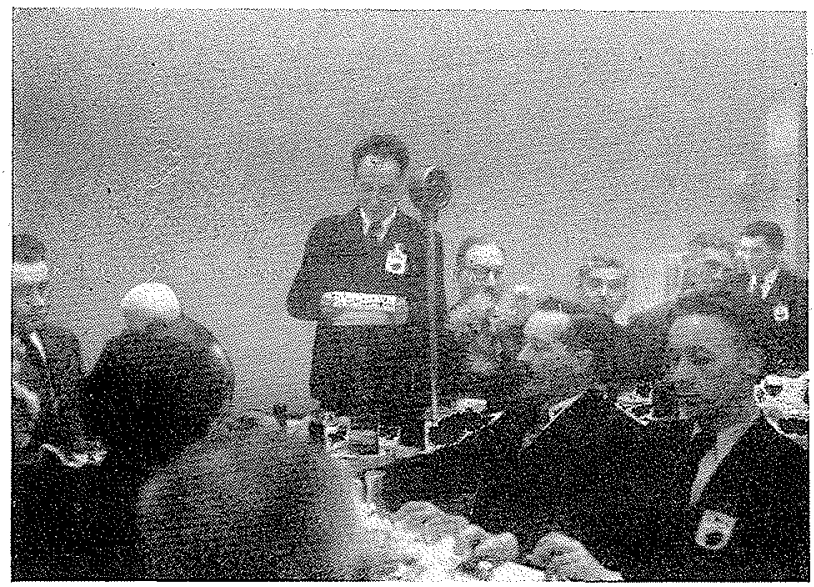

M. T. ESCCANGON, PIÉSIDENT DU C. Y. E. G. PRONONCANT SON ALIOCGTON AU COURS IDU BANQUET D'OUVERTURE

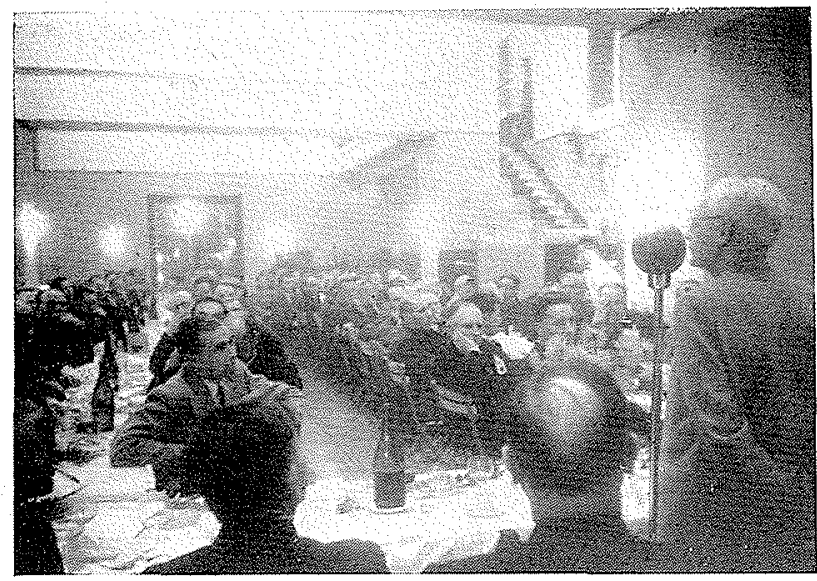

UNE VUE DU BANQUET D'OUVERTCRE lion électrique, qui prononca l'allocution de bienvenue aux 150 congressistes représentant treize pays étrangers, montrant la place importante que tient Grenoble dans une des branches particulièrement vivante de l'Industrie Nationale.

Après l'allocution de M. Hangelen, M. EsclanGON, Président du Conseil d'Administration du C.I.E.G. déclara le Congrès ouvert, et donna la parole au premier conférencier.

M. Hexky, de la Sochété Alais, Froges eT Camarave eT Progir entretint les congressistes de la question des «Diélectriques chlorés» et de leur cmploi comme huile isolante dans la construction des transformateurs. Il étudia le rôle de ces diélectriques, les spécifications qui leur sont imposées, les raisons qui ont amené les producteurs francais à se pencher sur ce problème, et les réalisations auxquelles ils ont abouti. Examinant successivement les différents produits mis au point et leurs caractéristiques, il montra les difficultés que i'emploi de ces produits nouveaux a soulevé et les solutions adoptées pour la construction des transformateurs. En conclusions, M. Hexry exposa les résultats obtenus et ce qu'il convient de penser de l'avenir des diélectriques chlorés.

Le soir, dans lè cadre du Téléférique, au-dessus de la mer de lumières de Grenoble, un grand banquet réunit les Autorités de la Ville, les Représentants des Sociétés ayant préparé le Congrès el les Techniciens.

La deuxième journce du Congrès, après une conférence de M. Latour, Ingénieur en chef aux Etrablissements Merlin et Gerin sur « Les essais en puissance des interrupteurs par rétablissement de tension amplifiée », conférence que nous pensons publier dans notre prochain numéro, fut consacrée à une visite d'usine et à une excursion en montagne.

Le matin, les congressistes visitèrent la Station d'essais à grande puissance des ETablissements Merin et Gerin. Au cours des événements qui ont précédé la Libération, cette Station d'essais, un des plus gros laboratoires d'essais en puissance existant en Europe, a été durement touchée. Aujourd'hui le laboratoire a non seulement été reconstruit dans un temps record, mais il a été perfectionné et agrandi.

Ses possibilités actuelles sont les suivantes :

$1^{\circ}$ ) en très haute tension, des essais jusqu’à $220 \mathrm{kv}$ et 4.500 mva grace à une intallation d"essais indirects ;

$2^{\circ}$ ) de 17,5 à $90 \mathrm{kv}$ : des essais directs par un transformateur élévateur jusqu'à $490 \mathrm{mva}$ et des essais indirects jusqu'à 2.500 mva. ;

$3^{\prime \prime}$ de 3 à $17,5 \mathrm{kv}$ : des essais directs sur l'alternateur jusqu'à 800 mva et des essais indirects jusqu'à 2.000 mva. ;

$4^{\circ}$ ) des essais basse tension permettant d'obtenir, par un transformateur abaisseur, $60.000 \mathrm{~A}$ sous $900 \mathrm{~V}$ et $250.000 \mathrm{~A}$ sous $250 \mathrm{~V}$. 
$\left.5^{\circ}\right)$ des essais à courant continu ou semi-continu jusqu'à: $100.000 \mathrm{~A}$;

$6^{\prime \prime}$ ) des essais surr onde de choc jusqu’à $2.000 \mathrm{kv}$ avec une énergie de 11,5 kilojoules;

$7^{\circ}$ ) des essais en puissance dírecte sur l"alternateur dans une atmosphère grisouteuse jusqu'à $17,5 \mathrm{kv}$.

L'après-midi, partis en autocars pour une excursion en montagne, les congressistes visitèrent tout d'abord la Centrale hydroélectrique de Champ-surDrac.

Cette chute dont l'aménagement remonte à 1901 a été réaménagée en 1941, non pas dans l'ensemble des ouvrages hydrauliques qui sont restés ce qu'ils étaient à l'origine avec simplement des adjonctions d'ordre secondaire, mais par la construction d'une nouvelle centrale avec groupe unique, alors que la précédente comportait six groupes.

L'équipement de cette chute comprend essentiellement :

- un barrage fixe établi sur le Drac prolongé côté rive droite par un ouvrage comportant deux vannes de chasse de $8 \mathrm{~m}$. d'ouverture;

- un ouvrage de prise d'une longueur de $80 \mathrm{~m}$., établi parallèlement au lit du Drac, et dans lequel sont aménagées 15 vannes de prise;

- un canal d'amenée d'une Iongueur de $600 \mathrm{~m}$. et d'une largeur variant de 22 à $80 \mathrm{~m}$. ;

- une chambre d'eau de $200 \mathrm{~m}^{2}$ et de $4 \mathrm{~m}$. de profondeur comportant un déversoir, des vannes de chasse, une grille à feuilles et une batterie de siphons susceptibles d'évacuer le débit total ;

- une conduite forcée de $3 \mathrm{~m} .30$ de diamètre, d'une longueur de $4.700 \mathrm{~m}$., partie en ciment armé, partie en tôle d'acier, comprenant trois cheminées dont deux établies sur son parcours et la troisième à l'extrémité ;

- la centrale équipée d'un groupe unique à axe vertical composé d'une turbine Francis de $9.430 \mathrm{cv}$ sous une chute nette de $28 \mathrm{~m}$., absorbant un débit de $28 \mathrm{~m} 3 / \mathrm{sec}$. à la vitesse de 250 tours/min., accouplée directement à un alternateur de $8.500 \mathrm{kva}$ sous $\cos \varphi 0,8$, soit $6.800 \mathrm{kw}$ à la tension de 10.000 volts (ce bâtiment abrite, outre les services auxiliaires et le tableau de commande, un papillon déchargeur capable d'évacuer dans le canal de fuite le débit total de la turbine en cas de fermeture de cette dernière;

- un poste extérieur de transformation et de couplage établi à proximité de la centrale comportant un transformateur de $8.500 \mathrm{kva}$ élevant la tension de 10.000 à $26.000 \mathrm{~V}$.

Le réaménagement de l'usine de Champ a permis de porter sa production annuelle à $48.500 \mathrm{kwh}$.

Après la visite de Champ-sur-Drac, le convoi passa à Vizille où, le 21 juillet 1788, une Assemblée provinciale, véritables Etats-Généraux du Dauphiné, prépara la Révolution Française.

Puis le convoi se divisa en deux groupes, l'un à destination du barrage du Sautet et de l'usine de Cordéac par la route Napoléon et les lacs de Laffrey, l'autre à destination du barrage du Chambon et

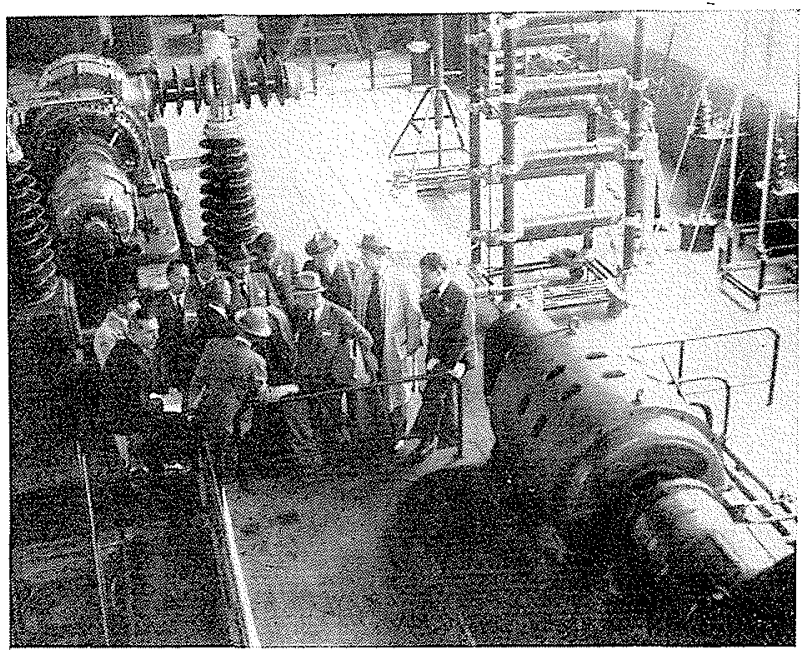

VISITE DES ETABLISSEMENTS MERLIN ET GERIN

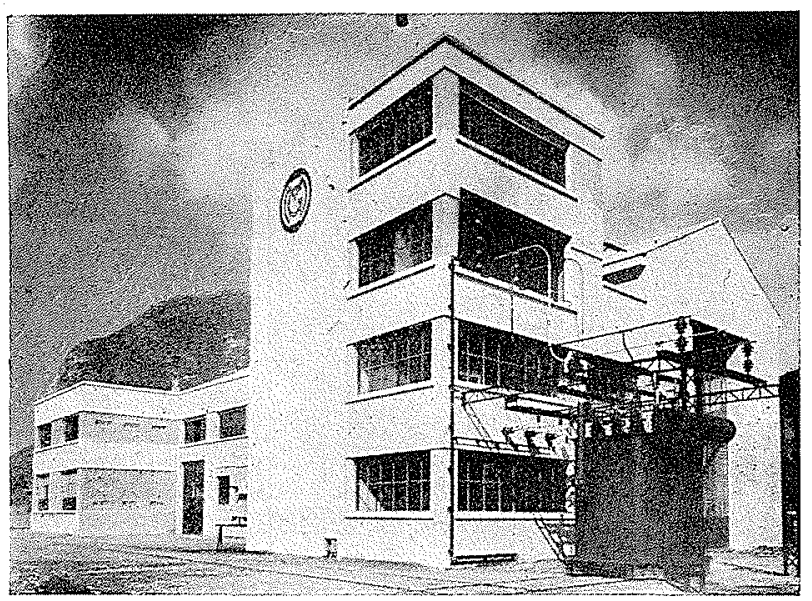

STATION D'ESSAIS DES ETABLISSEMENTS MERLIN ET GERIN

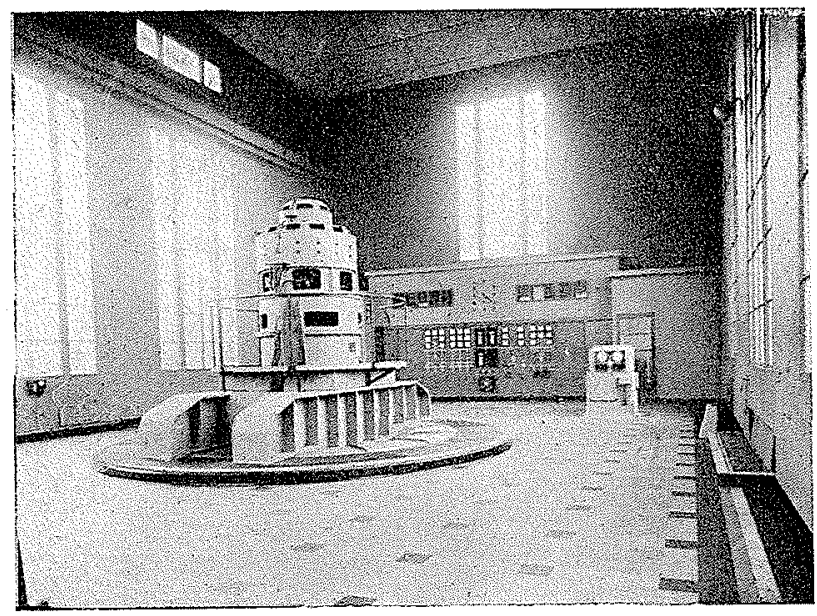

CHAMP-SULT-DRAC 
de l'usine de Pont-Escoffier sur le Vénéon, par la route des Alpes.

Mis en exploitation en 1935, le barrage du Sautet, utilisant le canion du Drac particulièrement étroit à cet endroit, est un barrage voûte qui présente les caractéristiques suivantes :

Hauteur, $126 \mathrm{~m}$.

Longueur de crête, $80 \mathrm{~m}$.

Volume total de maconnerie, $100.000 \mathrm{~m}^{3}$.

La superficie du bassin versant du Drac au Sautet est de $990 \mathrm{~km}^{2}$ et le réservoir a une capacité de 130.000 .000 de $m^{3}$ d'eau.

L'évacuation des crues est assurée par deux galeries de $350 \mathrm{~m}$. de long et $35 \mathrm{~m}^{2}$ de section, établies en souterrain sous la rive droite et donnant, lorsque le plan d'eau atteint la crête du barrage, un débit total de $1.400 \mathrm{~m}$.

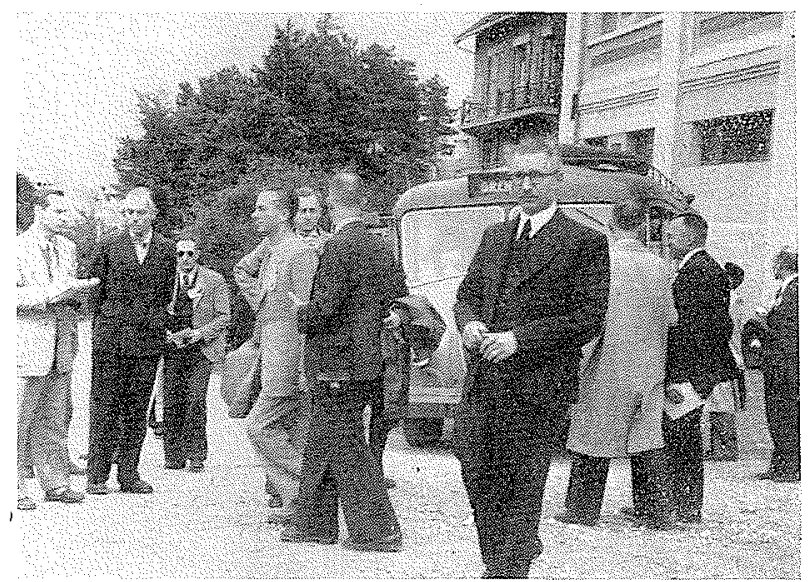

ARUTVER DES CONGRESSISTES AU SAUTET

La sécurité donnée par ce chiffre élevé est encore augmentée par l'aménagement de la crête du barrage qui pourrait déverser, au cas où une crue calastrophique dépasserait les prévisions.

L'usine, située en travers de la gorge, en partie sur un pont, en partie dans un souterrain de $26 \mathrm{~m}$. de profondeur creusé sous la rive droite est constituée par une voûte de $36 \mathrm{~m}$. 50 de portée.

L'équipement hydroélectrique comprend :

- 6 groupes principaux à axe horizontal comportant chacun deux turbines Francis d'une puissance ensemble de $16.000 \mathrm{cv}$ sous $93 \mathrm{~m}$. de chute, absorbant un débit total de $15 \mathrm{~m}^{3}$;

- 1 alternateur de $13.250 \mathrm{kva}, \cos \varphi=0,85$.

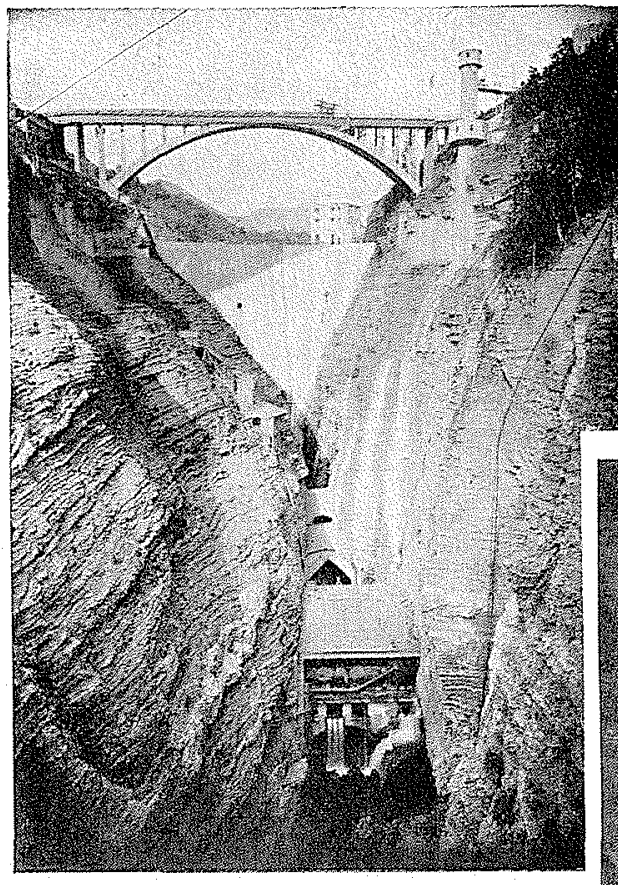

- 2 groupes auxiliaires de 330 kva portent la puissance de cet équipement aux environs de $80.000 \mathrm{kva}$.

Après avoir visité l'installation du Sautet, le premier groupe de congressistes, remontant en car, se dirigea, par des routes étroites, vers la gorge de Cordéac.

Cordéac «Fille du Sautet » fonctionne en série avec l'usine visitée précédemment. Le débit des deux usines, qui doit être le même, est régularisé par le réservoir du Sautet. Et l'on peut dire que Cordéac agit comme une succursale.

L'origine de la galerie d'amenće souterraine de $5 \mathrm{~m} .20$ de diamètre est placée sous l'usine du Sautet, dans son axe
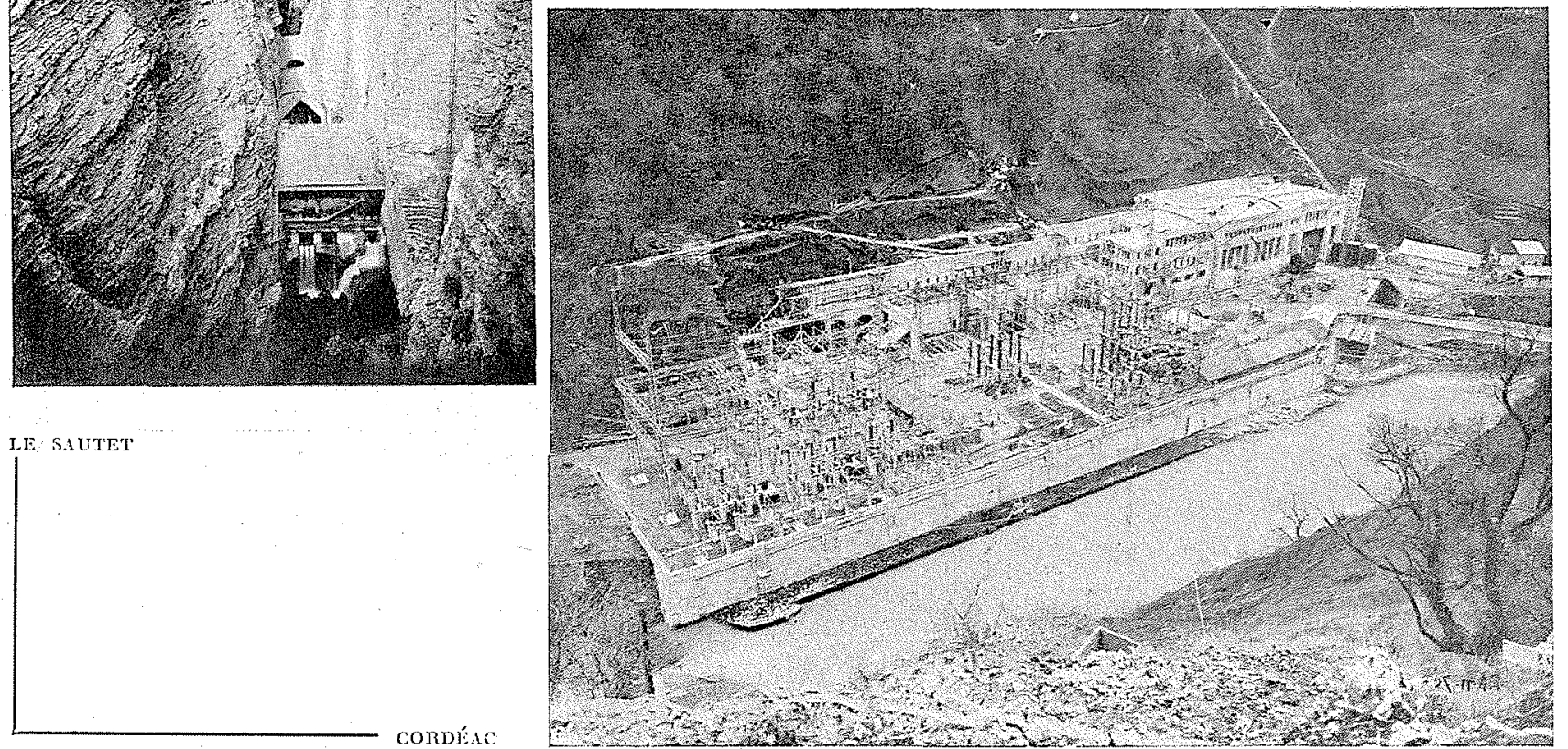
longitudinal. A partir de l'emplacement du batardeau d'entrée, son diamètre est de $5 \mathrm{~m} .80$

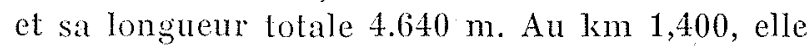
traverse Je Drac. Le pont, formé par la conduite elle-même est réalisé en béton fortement armé di soutenu par deux doubles supports en $\mathrm{V}$.

Un contre-barrage du type déversant situé à $250 \mathrm{~m}$. en aval du grand barrage constitue une retenue de $60.000 \mathrm{~m}^{3}$ et oblige les eaux évacuées par les turbines du Sautet à pénétrer dans la galerie d'amenée. Les caractéristiques de ce barrage sont les suivantes :

Hauteur $33 \mathrm{~m} .50$

Longueur (en doucine), $28 \mathrm{~m}$.

Longueur du déversoir en crête, $16 \mathrm{~m}$.

Volume de maconnerie, $9.000 \mathrm{~m}^{3}$.

L'extrémité de la conduite d'amenée débouche à la base d'une cheminće d'équilibre. L'usine génératrice comporte deux groupes hydroélectriques à axe vertical de puissance double l'un de l'autre. Les turbines, du type Francis, ont pour caractéristiques respectives :

GRAND GROUPE PETIT GROUP Puissance ....... $56.000 \mathrm{cv} \quad 28.000 \mathrm{cv}$ Débit maximum absorbé $58 \mathrm{~m} 3 / \mathrm{sec} .29 \mathrm{~m} 3 / \mathrm{sec}$. Vitesse .......... $214 \mathrm{t} / \mathrm{min} .300 \mathrm{t} / \mathrm{min}$.

Les alternateurs ont une puissance apparente de 42.000 et 21.000 kva sous une tension de $10,5 \mathrm{kv}$. L'usine de Cordéac n'est pas encore lotalement achevée, c'est donc un chantie'r que les congressistes ont visité. Cependant l'installation du groupe de $28.000 \mathrm{cv}$ étant terminée, la caravane a pu le voir fonctionner en essai.

Tandis que le premier groupe visitait l'aménagement du Drac et passait la soirée au mess des Ingénieurs de Cordeac, le second remontant la vallée de la Romanche voyait au passage les installations d'Electrochimie de la Sociétè Alais, Froges et Camargue, de Geller-Leleux, d'Ugne, de la Compagnie Francaise d'Acétrlìne. Toute cette vallée industrielle, aménagée de lonsue date avec de petits barrages au fil de l'eau dépend du réservoir du Chambon.

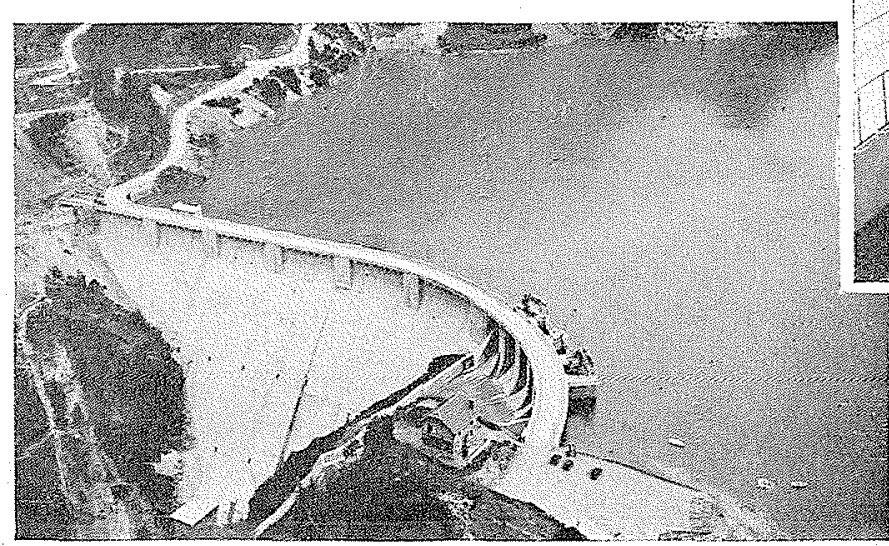

Le barrage du Chambon, où la caravane de cars et de voitures arliva vers $16 \mathrm{~h} .30$ a été mis en exploitation en 1944. Ciest un barrage du type gravité qui possède les caractéristiques suivantes :

Hauteur du lit : 90 mètres.

Hauteur sur fondations : 135 mètres.

Epaisseur en crête : 5 mètres.

Epaisseur à la base : 70 mètres.

Fruit amont : $5 \%$.

Fruit aval : $70 \%$.

Développement en crête : 290 mètres.

Sa construction a nécessité $300.000 \mathrm{~m}^{3}$ de béton. Le bassin versant a une superficie de 254 kilomètres carrés. Le débit moyen naturel de la Romanche est de $8,8 \mathrm{~m} 3 / \mathrm{sec}$; le débit d'étiage $1,5 \mathrm{~m}^{3} / \mathrm{sec}$, et la plus grande crue connue $70 \mathrm{~m}^{3} / \mathrm{sec}$.

La réserve utile est de 50.000 .000 de $\mathrm{m}^{3}$, la hauteur de la franche utile étant $60 \mathrm{~m}$. et le niveau de la retenue maximum 1.040 .

L'évacuation des crues, capable d'un débit de $400 \mathrm{~m} 3 / \mathrm{sec}$, est assurée par la levée de quatre vannes-secteur de $8 \mathrm{~m}$. de longueur et $3 \mathrm{~m} .50$ de hauteur.

L'usine de pied ou usine du Chambon, fonctionnant sous chute variable produit $25 \mathrm{M}$. kwh en année moyenne, celle de Saint-Guillerme, un peu en avant donne $160 \mathrm{M}$, kvh.

Après avoir admiré le lac du Chambon qui recouvre le village du même nom, les congressistes redescendant vers la plaine du Bourgd'Oisans s'engagèrent dans la vallée du Vénéon pour aller visiter l'aménagement de la chute de ce cours d'eau.

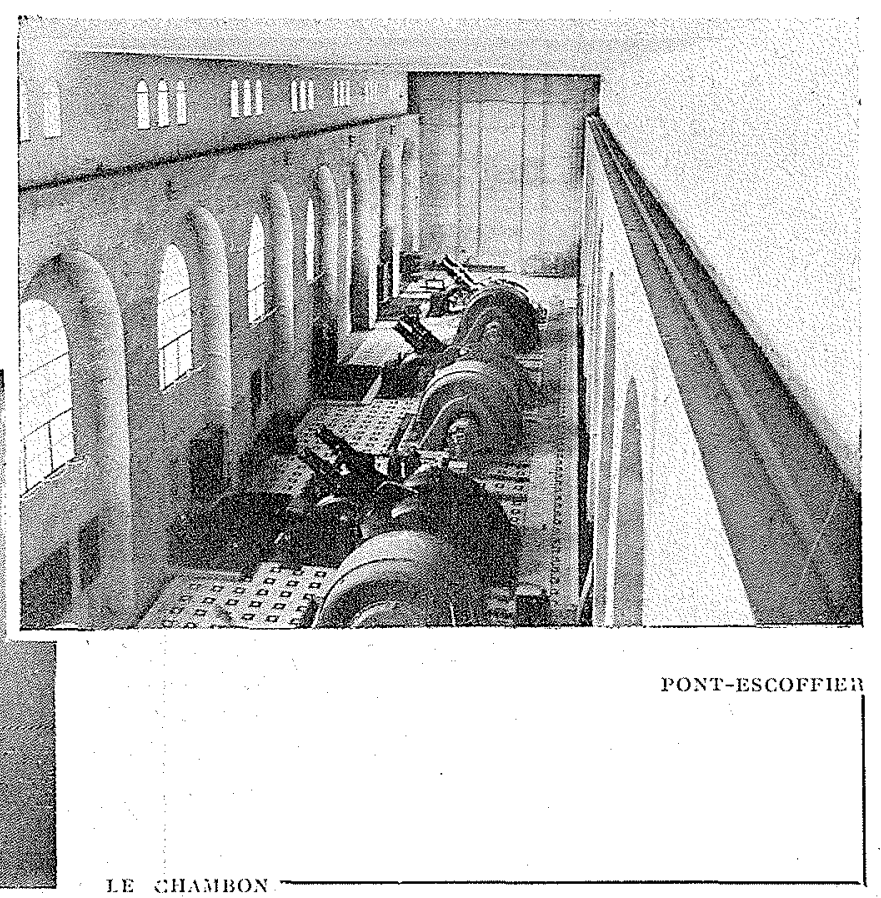




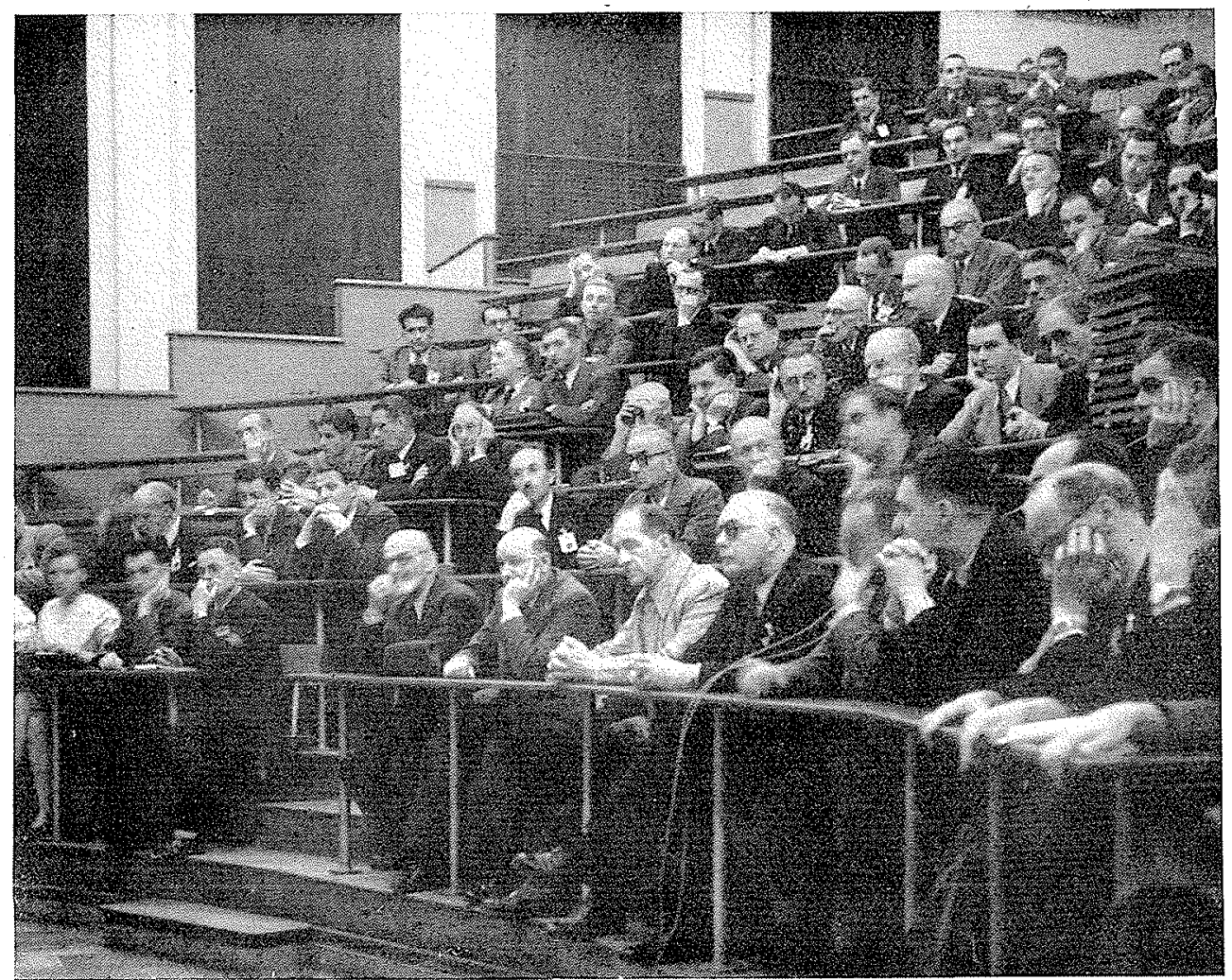

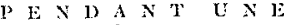

CON I E H E

de 10.400 et 13.500 kva, et quatre transformateurs triphasés de 27.500 kva.

Des travaux en cours complèteront en 1947 l'aménagement de la chute du Vénéon par l'apport des torrents de la Muzelle et du Lovitel, captés en haute montagne.

Un repas servi dans le cadre du Château de la Muzelle termina cette journée consacrée aux visites d"ins. tallations.

Le mercredi 10 juillet est une journée de travail. Dès 9 heures 30 , les congressistes s e retrouvent dans le grand amphi-

La chute du Vénéon, mise en expioitation en 1944, est caractérisée par deux ouvrages nettement distincts :

Le harrage de prise de Plan du Lac et la centrale de Pont-Escoffier reliés par une galerie d'amenée de $6.606 \mathrm{~m}$. sous la montagne, et une conduite forcée en tuyaux auto-frettés de $632 \mathrm{~m}$. de longueur et de $16 \mathrm{~m} 3 / \mathrm{sec}$. de débit.

Le bassin versant a une superficie totale de $250 \mathrm{~km}^{2}$. Le débit moven naturel du Vénéon est de $11,77 \mathrm{~m}^{3} / \mathrm{sec}$, le débit d'étiage $1,42 \mathrm{~m} 3 / \mathrm{sec}$., et la plus grande crue connue de $72 \mathrm{~m}^{3} / \mathrm{sec}$.

La réserve utile est de $\mathbf{1 5 . 5 0 0 . 0 0 0 \mathrm { m } ^ { 3 }}$ et la chute est équipée pour $16 \mathrm{~m}^{3} / \mathrm{sec}$. sous $400 \mathrm{~m}$. de chute brute, la puissance installée étant de $72.000 \mathrm{cv}$.

La prise d'eau comprend essentiellement trois parties :

- un barrage fixe faisant déversoir, d'une longueur de $20 \mathrm{~m}$. ;

- un barrage mobile composé de 4 vannes-secteur ;

- un dessableur.

L'usine de Pont-Escoffier est installée avec quatre turbines Pelton doubles ( 2 roules et 2 injecteurs par roue), d'une puissance unitaire de $18.000 \mathrm{cv}$ avec une vitesse de $600 \mathrm{t} . / \mathrm{min}$. Ell comporte également quatre altemateurs

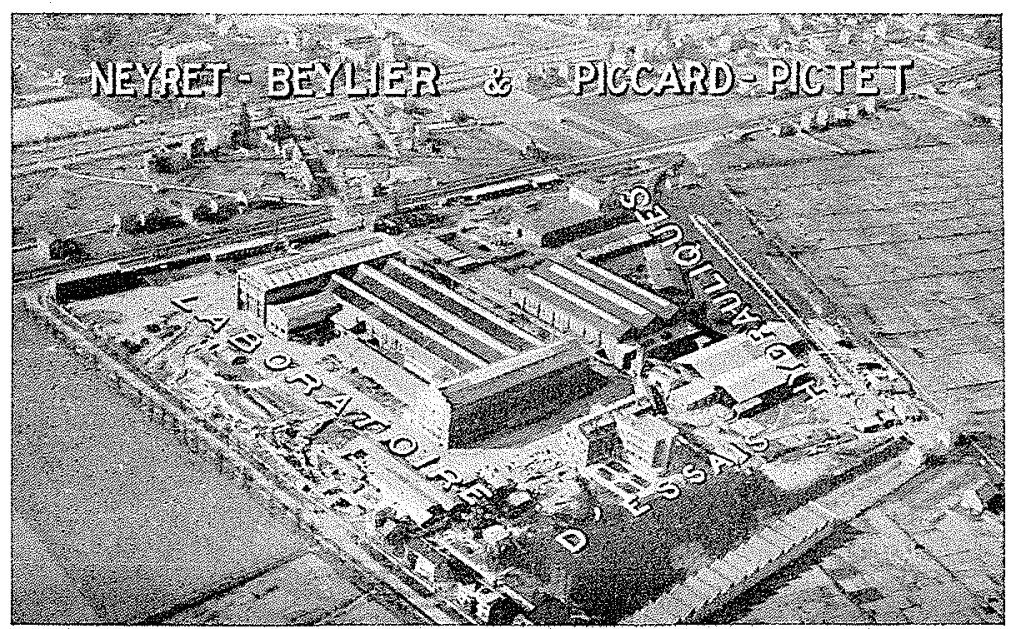


l'existence d'un Institut d'Electrochimie et Méta?lurgie dont les fonctions sont à peu près les mêmes, mais qui, étant Institut de Faculté, est indépendant de l'Institut Polytechnique.

Après avoir retracé l'activité de l'Institut Polytechnique : enseignement, laboratoires industriels, recherches scientifiques et ses relations avec la Faculté des Sciences, M. Esclangon passa la parole à M. Fontur, Professeur à la Sorbonne, ancien Sous-Directeur de l'Institut Polytechnique qui, dans une conférence que nous publions dans ce numéro, indiqua les difficultés de la définition de la «perte de charge».

MM. Neel, Professeur à la Faculté des Sciences et Writ, Maître de Conférences, Professeur à l'Institut Polytechnique décrirent ensuite un nou. veau type d'aimants permanents, réalisés sur le plan industriel, et dans le cadre de cet exposé, M. Felici, Maître de Recherches parla des applications nouvelles de l'électricité dans le domaine agricole, en particulier de l'utilisation des machines électrostatiques. Ces conférences dont nous avons pu apprécier tout l'intérêt, seront publiées dans un prochain numéro de la « Houille Blanche. »

M. Gignoux, de l'Académie des Sciences, Professeur à la Faculté des Sciences et à l'Ecole des Ingénieurs hydrauliciens traita des nappes souterraines profondes dans les vallées alpines et de leur importance du point de vue des aménagements hydroćlectriques. Cet exposé est publić dans notre présent numéro.

M. PAindé, Professeur à la Faculté des Sciences, des Lettres et à l'Ecole des Ingénieurs hydrauliciens termina la série de ces conférences par un bref exposé sur la sécheresse des années 1941-1946.

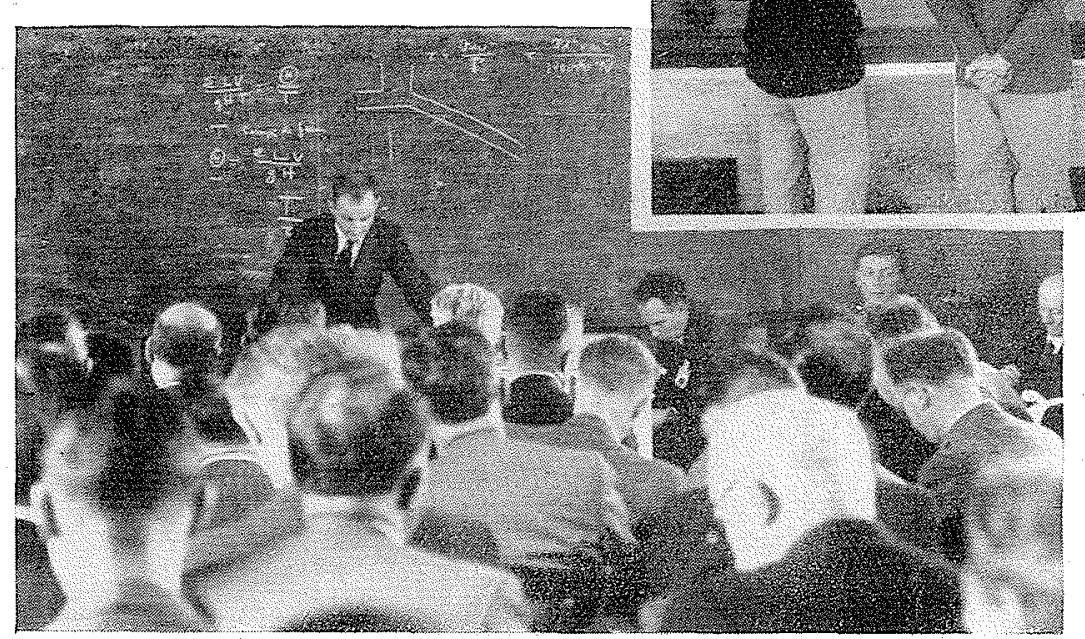

CONFEMENCE DE M. AWMERAS
M. P. Danal, Ingénieur en Chef du Service Essais et Recherches des Ateumers NeyreTBeylien el Piccard-Pictet, Directeur du Laboratoire d'hydraulique, devait présenter divers appareils à transformations conformes applicables aux tracés d'écoulements. Un malencontreux retard des services aériens d'Amérique du Nord nous priva de sa présence.

Après ces quelques exposés, une visite des Inslituls Polytechniques et Fourier termina cette matinée de travail.

L'après-midi était consacrée à la visite des Atelders Neyret-Beylater et Piccard-Pictet, spécialistes des Etudes sur modèles réduits et constructeurs de turbines. M. M. Garuen, Directeur Général des Ateliers N.-B.-P.-P. souhaita la bienvenue aux congressistes à Beauvert et, en quelques mots, retraca l'activité de la Station d'Essais avant que commençat la visite.

Depuis sa fondation en 1920, la Station d'Essais et Recherches des Ateliers N.B.P.P. a une activité sans cesse croissante et des problèmes de plus en plus complexes sont abordés.

Successivement, elle a étudié les évacuations de crues et les galeries de dérivation des barrages du Sautet, du Chambon, de Castillon, en
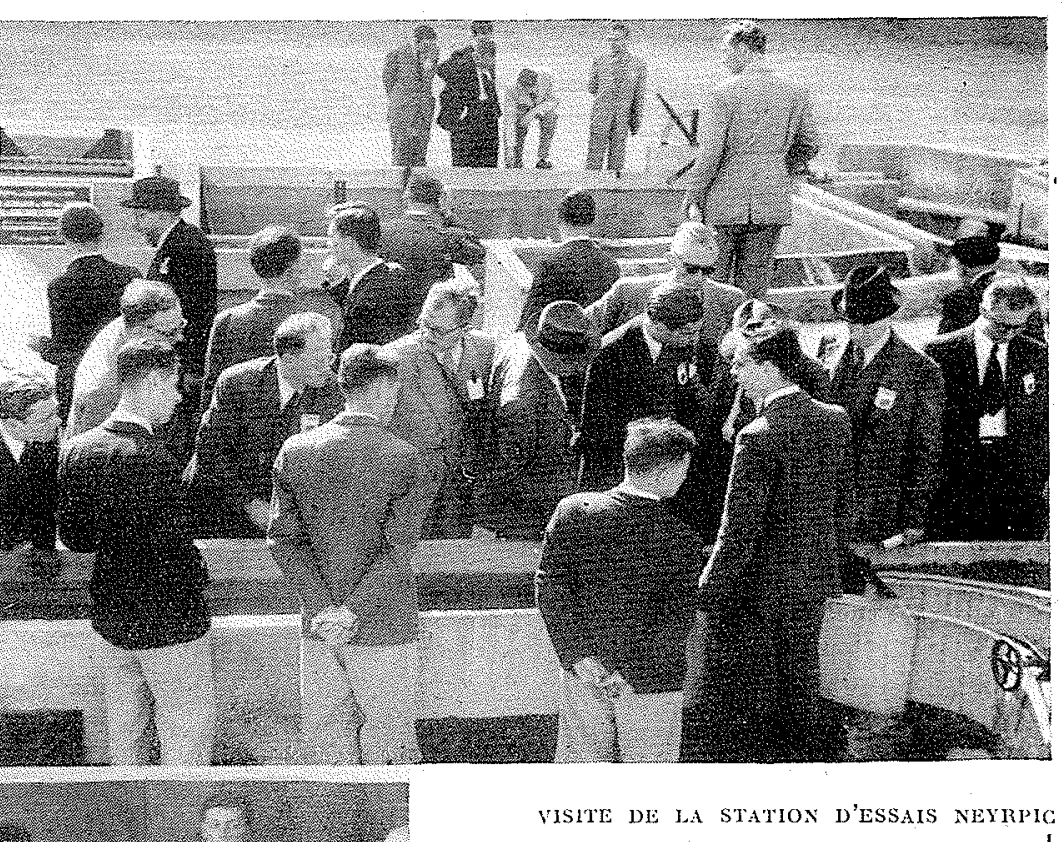


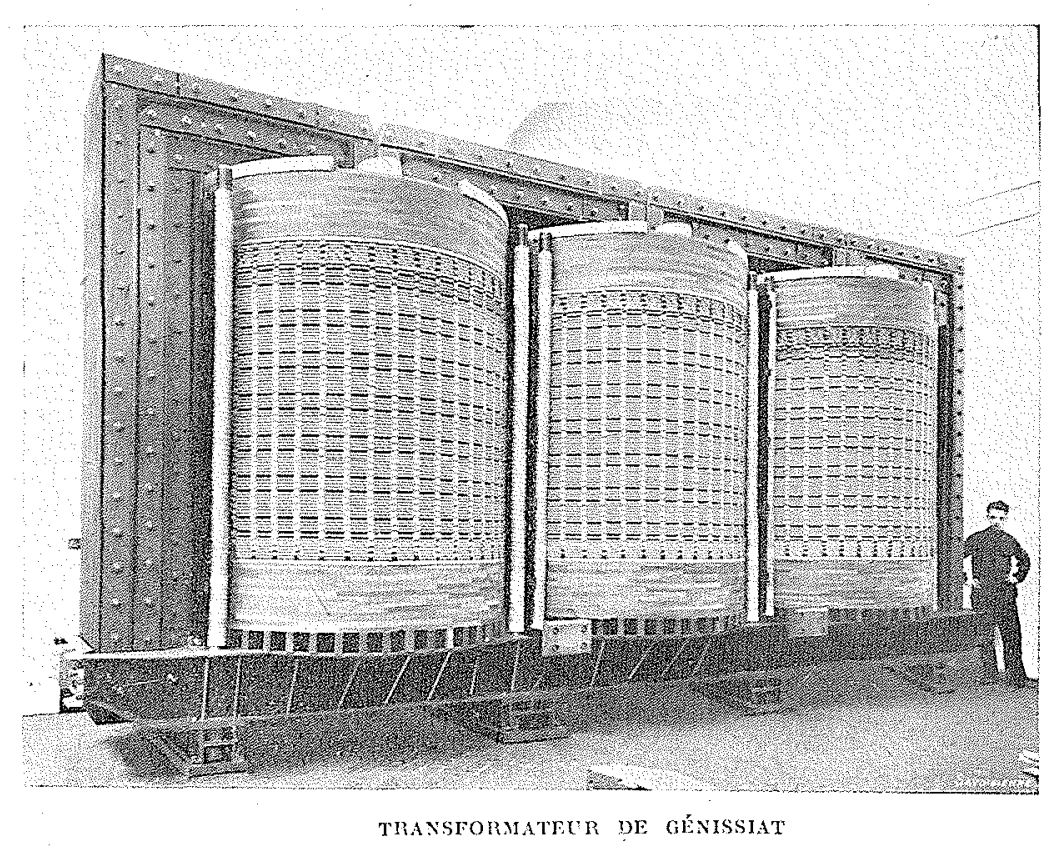

Signalons, cependant, que M. Gabes, Directeur du Bureau d'Etudes du Malériel hydraulique Alsthom-Charmilles publie dans ce numéro queiques remarques sur l'étude de M. Arméras.

La visite de Ia Station d'Essais clótura cette journée, essentiellement consacrée au travail.

Le jeudi 11 juillet, un départ matinal en cars et en voitures particulières permit aux congressistes d'aadmirer les beautés de la chaine de Belledonne, de la vallée du Grésivaudan, estompees dans une brume légère.

Le but initial de cette excursion étail la visite de la Société Savoisienne de Constructions Electriques à Aixles-Bains.

La Société Savoisienne de Constructions Electriques fondée en 1921, s'est spécialisée dans la fabrication des transformateurs et condensa-

France; de Bou-Hanifia, Hamiz, Zardezas en Afrique du Nord; de Pego do Altar, Castello do Bode, Nondego au Portugal.

Un champ très étendu de recherches est abordé avec les barrages en rivières, les engravements, et en général, tous les problèmes d'hydraulique fluviale à rectification de lit, coupures de boucles, etc.

En 1939, le poids de matériaux nécessaires à la coupure du Rhône à Génissiat est prévu avec une erreur inférieure à $3 \%$.

Parallèlement, une technique d'irrigation est mise au point et des réseaux entiers sont équipés en Algérie et en Argentine.

En 1944, sur un modèle de $450 \mathrm{~m}$. de longueur les problèmes hydrauliques posés par la traversée du Rhin par les Armées Alliées sont étudiés avec des résultats très satisfaisants.

Enfin, des études se poursuivent dans le domaine maritime: protection de rivages, aménagements de ports, utilisation de l'énergie thermique des mers, utilisation de la marée et de la houle.

Aucun domaine de l'Hydraulique ne reste ainsi inexploré.

Après l'allocution de M. Ganier, M. Arméras, Ingénieur à la Station d'Essais, prend la parole et parle de « l'étude de la stabilité des groupes hydroélectriques interconnectés, en tenant comple de l'inertie de l'eau dans les conduites forcées ». Cet exposé a fait la matière d'un article publié par la Houille Blanche $\left(n^{\circ s} 1\right.$, 2, 3) (1); nous ne reviendrons pas sur le sujet.

(1) La Houlle Blanche est à la disposition des persomnes n'ayant pas eu ees numéros, pour leur envoyer gracieusement un tiré à part de l'article de M. Almiras. teurs statiques de tous genres, toutes puissances et toutes tensions.

Au cour's de ses 25 premières années d'activité, elle a construit au total 55.000 transformateurs et 10.000 condensateurs représentant une puissance globale installée de 11.000 .000 de krva.

La Savoisienne s'est orientée de plus en plús vers l'exécution de très grosses unités el a livé notamment :

- A la centrale de la Truyère : 5 transformateurs de 33.000 kva et de 40.000 kva à 220.000 volts;

- Au poste de Kembs : 3 transformateurs de $31.000 \mathrm{kva}$ à 150.000 volts et 2 transformateurs de 62.000 kva à 220.000 volts

- A l'usine de l'Aigle : 2 transformateurs de 60.000 kva à 220.000 volts.

\section{VISITE DY LA SOCHETE SAYOISIENXE} DE CONSTIUCTIONS JELCTRIOHES

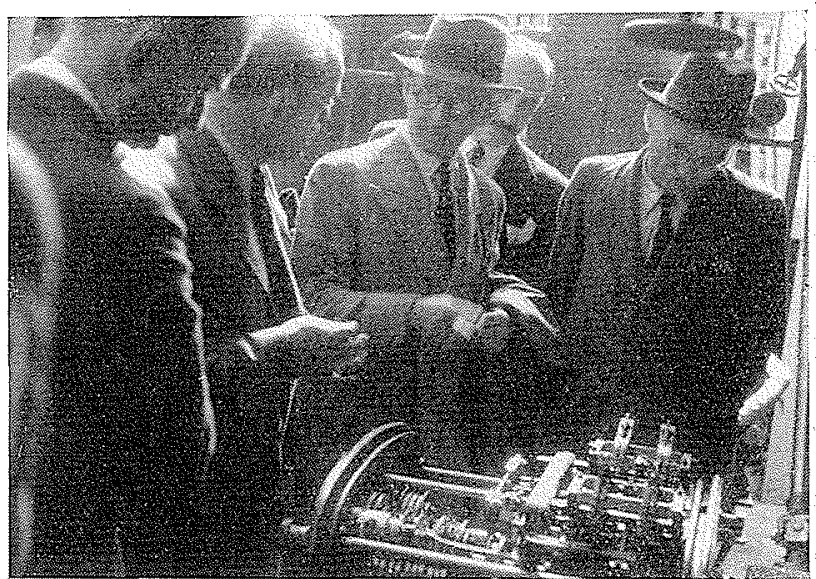


Au cours de la visite des ateliers, les congressistes onl pu voir, en cours de construction :

4 transformateurs de 52.500 kva pour la Hollande ;

4. transformateurs de 70.000 kva a 220.000 volts destinés a la centrale de Génissiat;

1 transformateur de $100.000 \mathrm{kva}$ à 3 enroulements $15.000 / 150.000 / 220.000$ volts avec réglage

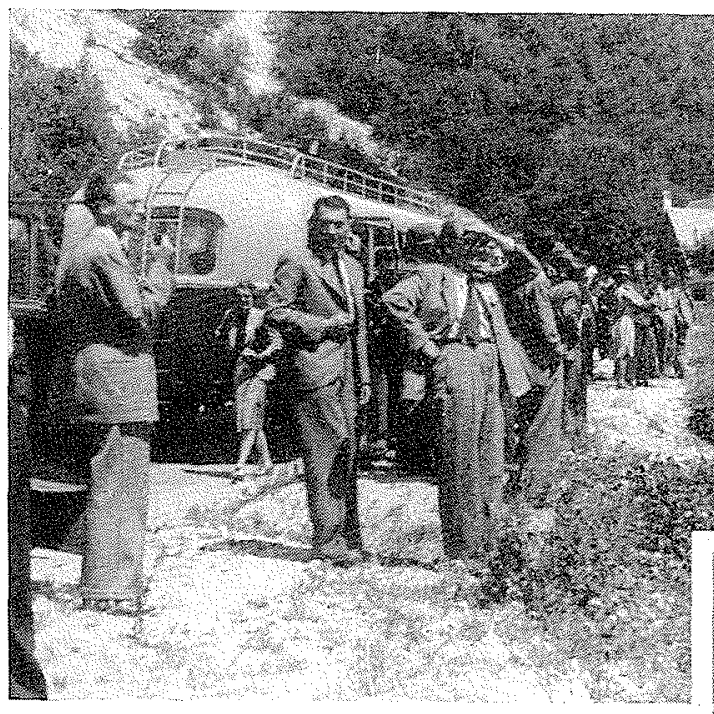

HAITE AVANT LE TUNNEL DU COL DU CHAT

de la tension en charge sur le cóté $150 \mathrm{kv}$, pour le poste de Mazingarbe dans le Nord.

Après la visite de la Savoisienne, les congressistes quitterent Aix-les-Bains et, par la route en corniche qui longe le lac du Bourget, atteignirent le col du Chat arant de s'enfoncer dans les collines qui mènent à Belley.

Belley, patrie de Brillat-Savarin, relais gastronomique réputé, les accueil. lit au déjeuner. Puis, sous un soleil éclatant, ce fut la descente vers Génissiat, le gros ouvre de la construction hydroélectrique francaise.

Le Rhône, en sortant du lac Léman, entre en France et s'enfonce presque tout de suite dans des gorges profondes à Fort-l'Ecluse, à environ une vingtaine de kilometres en amont de Génissiat. Ces gorges se prolongent jusque vers Seyssel.

Génissiat est la région la plus aval de ces gorges où les falaises rocheuses atteignent uno hauteur suffisante pour permettre de construire un barrage. C'est là une des plus fortes raisons qui a conduit à choisir le site de Génissiat.

Par ailleurs, les prospections géologiques ont montré que le rocher calcaire était de très bonne qualité et, d'autre part, la voie ferrée GenèveLyon coupe le chantier, ce qui est de nature à en faciliter grandement la desserte.

Le lac de Génissiat s'étendra jusqu'a la frontière Suisse. Il aura 23 kilomètres de longueur ct une capacité de 53.000 .000 de $\mathrm{m}^{3}$. Cette capacité est assez faible en comparaison du débit du Rhòne. Celui-ci, en efret, est déjà un très grand fleuve à Génissiat. Pour un étiage de $140 \mathrm{~m} .$, un débit moyen de $400 \mathrm{~m}^{3} / \mathrm{sec}$., ses crues ne sont cependant pas teliement élevées : elles atteignent $2.000 \mathrm{~m} 3 / \mathrm{sec}$. Ceci tient à l'effet de régulateur de crue du lac Léman.

L'aménagement de la chute de Génissiat, commencée en 1937, alimentera une usine de $420.000 \mathrm{kw}$ accolée au barrage. Cette usine comportera 6 turbo-alternateurs dont 4 de $70.000 \mathrm{kva}$ et 2 de 20.000 liva, ainsi que 2 groupes auxiliaires.

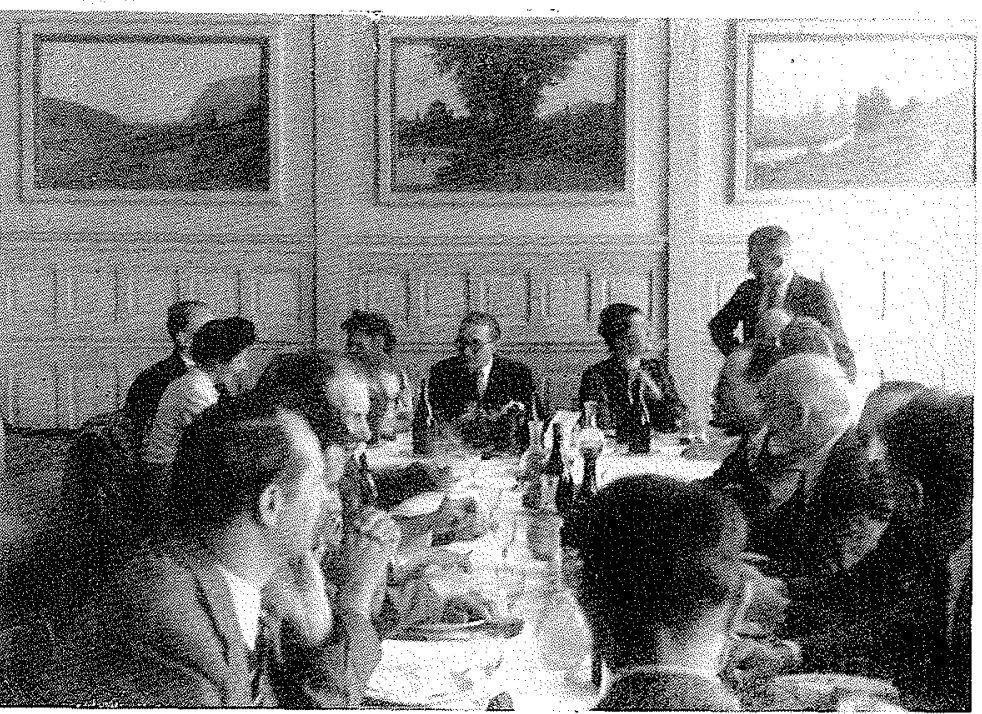

Eu égard à l'avancement des travaux et malgré les graves destructions de 1940, la mise en service du premier groupe est prévue pour la fin de l'année 1947.

La superficie du bassin versant de Génissiat est de $19.910 \mathrm{~km}^{2}$, le débit moyen $393 \mathrm{~m}^{3}$. La cote de retenue normale est à $330 \mathrm{~m}$. 70. La hauteur de chute brute entre 60 et $69 \mathrm{~m}$.

Le barrage en construction sera du type poids, incurvé en plan suivant un rayon de $500 \mathrm{~m}$. Sa hauteur maximum atteindra $104 \mathrm{~m}$., la longueur du couronnement $140 \mathrm{~m}$., sa largeur $9 \mathrm{~m}$.

L'évacuation des crues sera assurée par deux évacuateurs, un superficiel rive droite. $(2.700$ 
mètres-cubes/sec.), un souterrain de demi-fond $\left(1.300 \mathrm{~m}^{3} / \mathrm{sec}\right.$.).

Les prises d'eau au nombre de 6 auront un diamètre de $12 \mathrm{~m}$, les conduites forcées des groupes principaux munies de charpentes de renforcement, un diamètre de $5 \mathrm{~m}$. 75 , celles des groupes auxiliaires $1 \mathrm{~m} .10$.

L'usine sera installée pour une puissance annuelle moyenne de 1.550 .000 .000 de $\mathrm{kwh}$ avec quatre turbines Francis à axe vertical de $90.000 \mathrm{cv}$ sous $69 \mathrm{~m}$. et deux turbines Francis de $120.000 \mathrm{cr}$ sous $54 \mathrm{~m}$. Les altemateurs triphasés auront une puissance normale respective. de $65.000 \mathrm{kw}$ et $1.750 \mathrm{kw}$, et les transformateurs $70.000 \mathrm{kva}$ et $5.000 \mathrm{kva}$.

Après la visite totale du chantier bourdonnant d'activité - près de 3.000 ouvriers y travaillent actuellement - la caravane reprit la route d'Aix-les-Bains, où, après le dîner de clôture, M. Esclangon, Président du Conseil d'Administration du Congrès International de l'Electricité de Grenoble tira la conclusion de ces journées, remerciant les délégations étrangères d'être venues si nombreuses et souhaita de pouvoir, à nouveau, réunir un Congrès dans quelques années avec un programme d'organisation différent.

M. Pamler, Ingénieur, chef de service des Ateliers de Construclions Electriques de Charleroj remercia, au nom des Congressistes, disant leur reconnaissance pour l'accueil qu'ils avaient rencontré, louant l'organisation du Congrès qui contribua à dissiper la pensée que, du fait de la guerre, la France subissait un appauvrissement général.

M. Donkin, au nom des délégués de langue anglaise, remercia à son tour les organisateurs du C. I. E. G. et dit combien les congressistes avaient admiré ce pays qui après six années de guerre, commence à se relever avec une grande énergie.

Ce sera la conclusion que nous tirerons de ces journées, l'effort de travail du pays qui, actuellement, groupe toutes ses énergies pour refaire de la France, un pays fort et vivant.

R.-H. HoNorÉ.

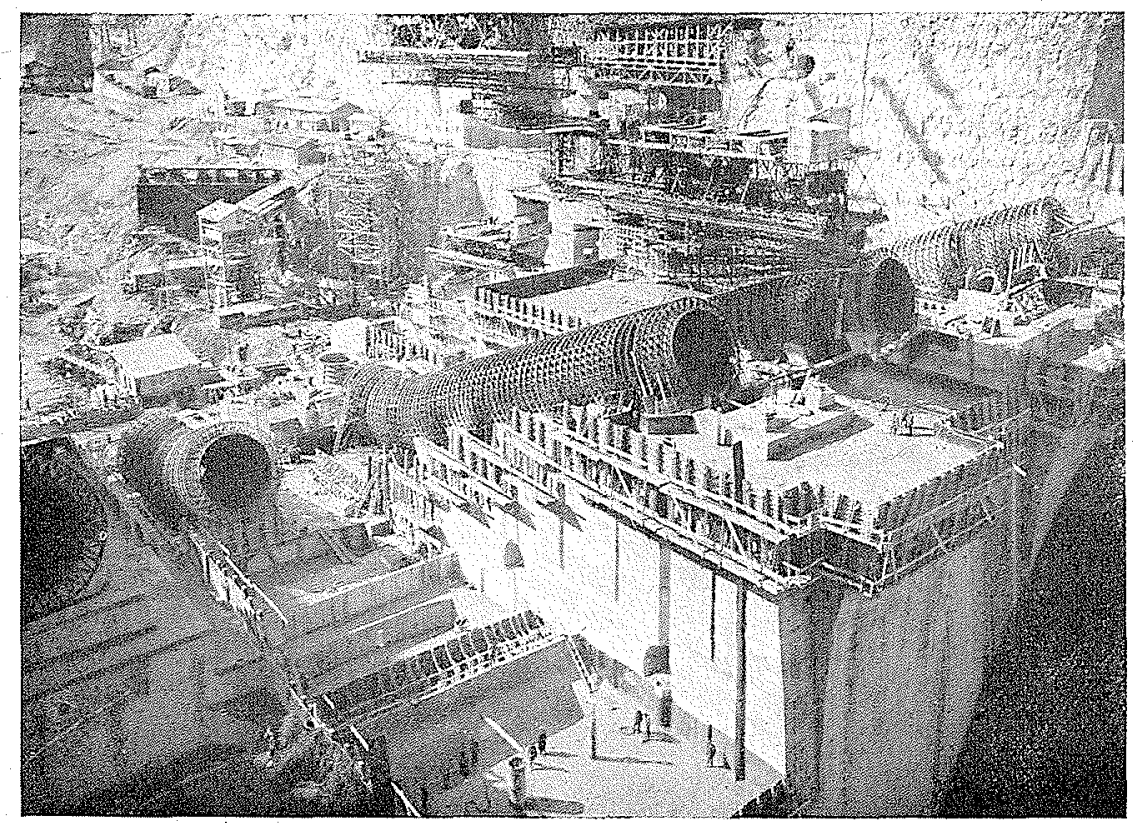

CHANTIER DE GENISSIAT 